\title{
Conséquences d'une utilisation extensive des prairies en production ovine
}

\author{
A Brelurut 1 , F Louault 2, M Benoit ${ }^{3}$ \\ ${ }^{\dagger}$ Adaptation des herbivores aux milleux ; ${ }^{2}$ Agronomie et mycologie ; ${ }^{3}$ Economie de l'élevage, INRA, \\ Theix, 63122 St-Genès-Champanelle, France
}

La diminution régulière des cours de la viande ovine et la disponibilité accrue en terres amènent certains éleveurs à recourir à l'extensification par agrandissement de leur exploitation. Mais les conséquences zootechniques, agronomiques et économiques d'une utilisation extensive du territoire sont mal connues. C'est pourquoi nous avons comparé les résultats techniques et économiques de 2 lots de 135 brebis limousines exploitant chacun pendant 4 années consécutives 17,4 ha (lot Témoin $\mathrm{T}$ ) et 24,5 ha (lot Agrandi A) de prairies. Les chargements correspondants s'élevaient à 1,17 et à $0,85 \cup G B / h a$. Les objectifs de production et la conduite de ces deux troupeaux étaient comparables : maintien d'un bon niveau de performances avec 1 agnelage/brebis/an (2/3 des mise bas au printemps avec production d'agneaux à l'herbe, et $1 / 3$ à l'automne avec production d'agneaux de bergerie). Dans les deux cas, le coût minimal de production a été recherché en favorisant le pâturage et en limitant les principaux intrants.

Les performances de reproduction ont été identiques entre les deux lots (181 et $178 \%$ de prolificité pour A et T, 160 et $150 \%$ de productivité numérique au sevrage), mais les carcasses des agneaux produites en A étaient un peu plus lourdes (15 kg vs 14,1 en $T$ ). Globalement, les brebis du lot $A$ ont donc été légèrement plus productives que celles du lot $\mathrm{T}(23,4 \mathrm{~kg}$ de carcasse/brebis/an vs $22,3 \mathrm{~kg})$.

L'agrandissement des surfaces a permis un nombre de journées de pâturage supérieur de $15 \%$, et une diminution importante des principaux intrants (engrais et aliments concentrés). En A, la fertilisation annuelle a été de $18 \mathrm{~kg}$ de $\mathrm{N} / \mathrm{ha}$, de $14 \mathrm{~kg}$ de $\mathrm{K}_{2} \mathrm{O}$, et de 6 $\mathrm{kg}$ de $\mathrm{P}_{2} \mathrm{O}_{5}$, contre 67,34 et $17 \mathrm{~kg}$ en $\mathrm{T}$. Seuls $38 \%$ de la surface fauchable de $A$ ont reçu de l'azote, contre $96 \%$ de celle de $B$. De même, la consommation d'aliments concentrés a été beaucoup plus faible en $A$ : $84 \mathrm{~kg}$ contre $117 \mathrm{~kg} / \mathrm{brebis}$ en $\mathrm{T}$. Cet écart est principalement dû à la conduite des brebis agnelant au printemps dont la mise à l'herbe a souvent été plus précoce (de 29 jours l'année la plus favorable). De plus, la plus grande surface disponible et la meilleure qualité des prairies pâturées grâce à une contribution importante du trèfle blanc ont permis de maintenir les performances de croissance à un niveau élevé (262 g/j entre 10-30 j, 279 entre 30-70 j pour les mâles allaités doubles au pâturage) tout en réduisant les apports totaux d'aliments concentrés jusqu'à l'abattage à 7,7 kg par agneau, contre $22,6 \mathrm{~kg}$ pour le lot $\mathrm{T}$.

Grâce au produit ovin plus important obtenu en $A(787 \mathrm{~F} /$ brebis/an contre $758 \mathrm{~F}$ pour $\mathrm{T})$ et aux charges proportionnelles plus faibles (281 F contre 317), le supplément de marge brute finale a atteint $97 \mathrm{~F} /$ brebis. Globalement et en moyenne sur 4 années, la marge brute de $\mathrm{A}$ a été supérieure de $23 \%$ à celle de T (59100 F contre $48100 \mathrm{~F}$ ), ce qui permettrait de couvrir un supplément maximal de charges de structures lié aux seules surfaces supplémentaires de $1540 \mathrm{~F} / \mathrm{ha}$, sans augmentation de cheptel, de bâtiments et de stocks.

En conclusion, cet agrandissement, accompagné d'une exploitation de la totalité des surfaces en herbe et d'une diminution raisonnée des intrants se traduit par des résultats techniques et économiques favorables. 